\title{
Does the Number of Available Strategies Change How Children Perform Cognitive Tasks? Insights from Arithmetic
}

\author{
Patrick Lemaire ${ }^{1}$, Koen Luwel $^{2} \&$ Fleur Brun ${ }^{1}$ \\ ${ }^{1}$ Aix-Marseille University, CNRS, LPC, Marseille, France \\ ${ }^{2}$ KU Leuven-Campus Brussels, Brussels, Belgium \\ Correspondence: Patrick Lemaire, Aix-Marseille University \& CNRS, 3, Place Victor Hugo, Case D, 13331, \\ Marseille, France. E-mail: patricklemaire13@gmail.com
}

Received: May 14, 2017

Accepted: June 8, 2017

Online Published: June 28, 2017

doi:10.5539/jedp.v7n2p43

URL: http://doi.org/10.5539/jedp.v7n2p43

\begin{abstract}
Fifth and seventh-graders accomplished computational estimation tasks in conditions where only one versus two strategies were available. Children were told which strategy to execute on each problem. Results showed that both groups of children were faster under one-strategy condition than under two-strategy condition and that age-related differences in performance were larger under two-strategy condition. Also, differences in strategy performance tended to vary as a function of the number of strategies, and this strategy difference was largest in younger children. These findings have implications to further our understanding of strategy execution in arithmetic and in other cognitive domains, as well as of age-related differences in children's performance during cognitive development.
\end{abstract}

Keywords: arithmetic, computational estimation, elementary-school children, strategies

\section{Introduction}

In many cognitive domains, children use a variety of strategies (Siegler, 2007). A strategy is "a procedure or a set of procedures to achieve a higher level goal" (Lemaire \& Reder, 1999, p. 365). Children's cognitive performance as well as age-related differences in this performance crucially depend on which strategies children use, how often they use each available strategy, and how efficient they are at selecting and executing strategies (see Siegler, 1996, for an overview). In this study, we focused on strategy execution. Theoretical and empirical works have shown that relative strategy difficulty depends on the number and type of procedures included within each strategy: Strategies including more and/or harder procedures yield longer latencies and higher error rates. Previous findings also revealed that age-related differences were largest when children use harder strategies than easier strategies and that relative strategy performance is influenced by situation, person, and problem characteristics (e.g., Siegler, 2007). Unknown is whether age-related differences in children's cognitive performance are influenced by the number of available strategies. The present experiment addressed this issue in the context of arithmetic problem solving.

When children are given either simple (e.g., $3 \times 4 ; 5+7)$ or more complex (e.g., 27-9; 38+46) arithmetic problems, they use several strategies. For example, in computational estimation tasks, investigated here, when children are asked to provide approximate sums to problems like 52+47, they use several rounding strategies, like rounding both operands down or up to their closest decades (e.g., 50+40,60+50), or like rounding one operand down and the other up to its closest decade (e.g., 50+50). These strategies are known and spontaneously used by children as young as seven years old, and different strategies yield different levels of speed and accuracy (e.g., LeFevre, Greenham, \& Waheed, 1993). For example, rounding both operands down is faster and more accurate (i.e., estimated and correct sums are closer) on small-unit problems (i.e., problems with sum of unit digits $<10$, like $41+62$ ) and rounding both operands up is most efficient on large-unit problems (i.e., problems with sum of unit digits $>10$, like $37+49$ ). Relative strategy performance has been found to vary not only with problem features, but also with children's characteristics (e.g., age, arithmetic skills) and with situational demands like the need to respond quickly and/or accurately (e.g., Dowker, 1997; Dowker, Flod, Griffiths, Harriss, \& Hook, 1996; LeFevre et al., 1993; Lemaire \& Lecacheur, 2002; Reys, Rybolt, Bestgen, \& Wyatt, 1982; Sowder \& Markovits, 1990). For example, Lemaire and Brun (2014; see also Lemaire \& Lecacheur, 2011) found that when children 
accomplished computational estimation tasks, young children executed the rounding-down strategy more quickly on small-unit problems (i.e., doing $30+40$ to estimate $32+46$ ) than on large-unit problems (i.e., doing $20+30$ to estimate 24+39), and that this Strategy x Problem interaction was stronger in third than in fifth graders. Similarly, Lemaire and Brun (2014) found that age-related differences in latencies to execute the rounding-down and the rounding-up strategies were larger under short response-stimulus interval condition (i.e., when the next problem was displayed $900 \mathrm{~ms}$ after participants' answer) than under longer response-stimulus interval condition (i.e., the next problem was displayed $1900 \mathrm{~ms}$ after participants' answer). In other words, both age-related differences and relative strategy performance vary as a function of problem type and situational characteristics. Unknown however is whether age- and strategy-related differences are influenced by the number of strategies that children use.

The goal of the present experiment was to determine whether effects of children's age and of strategies on cognitive performance are influenced by how many strategies are used. This is important as it would have a number of significant implications to further our understanding of both strategic behaviors and age-related differences in performance during children's cognitive development. For example, one of the important theoretical implications concerns determiners of relative strategy performance. Computational models of strategies (Lovett and Anderson (1996)'s ACT-R model; Lovett and Schunn (1999)'s RCCL model; Payne, Bettman, and Johnson (1993)'s adaptive decision maker model; Rieskamp and Otto (2006)'s SSL model; and Siegler and Arraya (2005)'s SCADS* model; see overview by Marewski and Link, 2014) share the core assumption that relative strategy performance depends on the number and types of mental procedures involved in each strategy. That is, individuals are faster with strategies that involve fewer and/or easier procedures. Finding that relative strategy performance differs in conditions where children use one versus two strategies would suggest that strategy execution is also influenced by some contextual factors, like the number of strategies brought to the task, above and beyond the component processes of each strategy.

From a developmental perspective, in most cognitive domains, age-related differences in children's cognitive performance are larger for harder than for easier strategies. For example, in arithmetic, many researchers found that age-related differences in performance are larger when children use harder, counting strategies relative to when they use an easier, direct retrieval strategy to solve arithmetic problems like $8+4$ (see Cohen-Kadosh \& Dowker, 2015; Geary, 1994, for overviews). Finding that these age-related differences for easier and harder strategies depend on the number of strategies that are used would imply that age-related improvements in children's performance are not only the result of increased efficacy at executing strategies but also at managing multiple strategies.

In the present study, fifth and seventh graders were asked to find sum estimates to two-digit addition problems. For each problem, a cue indicated which strategy children had to execute, which means that children did not have to select a strategy themselves. We controlled strategy selection to assess the role of the number of strategies on children's performance. By doing so, we avoided that strategy execution would be contaminated by differences in the frequency with which children would use each of the available strategies as well as differences in the type of problems on which the respective strategies would be applied. Children were tested under both a two-strategy and a one-strategy condition. In the two-strategy condition, children solved a set of 32 problems and were asked to execute either a mixed rounding-down or a mixed rounding-up strategy on each problem. In the Mixed Rounding-Down strategy (MRD), children rounded the first operand down and the second operand up to the closest decades (e.g., doing 40+70 to estimate 43+68). In the Mixed Rounding-Up strategy (MRU), children rounded the first operand up and the second operand down to the closest decades (e.g., doing 50+60 to estimate $43+68$ ). In the one-strategy condition, children were asked to solve a first set of 16 problems and were cued with the same strategy on all these 16 problems. Children were also asked to solve another set of 16 problems and were cued with the other strategy on all these 16 problems. MRD and MRU were tested because previous works showed that both fifth and seventh graders know and spontaneously use these two mixed-rounding strategies (LeFevre et al., 1993), and because previous works on relative strategy execution found that MRD is easier than MRU and yields better performance (e.g., Hinault, Lemaire, \& Phillips, 2016; Lemaire \& Brun, 2014; Lemaire \& Hinault, 2014; Uittenhove \& Lemaire, 2012).

As previous works found that the number of strategies individuals use to accomplish a given task is related to their executive functions (Ardiale, Hodzik, \& Lemaire, 2012; Hodzik \& Lemaire, 2011), we hypothesized that the number of available strategies would influence children's performance and age-related differences in this performance. We therefore tested the following predictions. First, children were expected to be slower and less accurate in the two- than in the one-strategy condition. This could happen if children have to use more executive control resources in the two-strategy condition relative to the one-strategy condition to maintain both strategies 
activated in working memory and to switch between strategies across problems. Second, if the number of strategies affects strategy execution, relative strategy performance should differ in the one- and two-strategy conditions, such that speed and accuracy should decrease more strongly for the harder (MRU) strategy than for the easier (MRD) strategy from the one- to the two-strategy condition. Again, this should occur if managing two strategies requires more resources than managing only one strategy and because children need more resources to execute the harder than the easier strategy. Third, larger age-related differences were expected in the two-strategy condition relative to the one-strategy condition. This would happen if the two-strategy condition requires more processing resources that are known to increase with children's age (Lemaire \& Lecacheur, 2011). Finally, a Group x Number of strategies x (MRD/MRU) Strategy interaction was expected if, relative to older children, younger children are more influenced by the number of strategies while executing the harder strategy than when using the easier strategy.

\section{Method}

\subsection{Participants}

Seventy-two children were tested: 36 fifth graders (17 girls; mean age=130 months; range=123-144) who came from one elementary school and 36 seventh graders ( 13 girls; mean age $=153$ months; range $=145-165)$ who were drawn from two secondary schools located in Flanders, the Dutch-speaking part of Belgium. Children's parents provided written informed consent, and children were told that they could quit the experiment at any moment.

\subsection{Stimuli}

Two sets of 16 two-digit addition problems (e.g., 24+39) each were selected for the two-strategy condition. All problems included one operand with its unit digit smaller than five and the other operand with its unit digit larger than five. In each set of 16 problems, the unit digit of the first operand was smaller than five and the unit digit of second operand was larger than five on half the problems (and the reverse for the other problems). Both sets of 16 problems were matched on the size of correct sums and on percent deviations between estimates and correct sums.

Moreover, following previous findings in arithmetic (see Campbell, 2005; Cohen Kadosh \& Dowker, 2015, for overviews), the following factors were controlled: (a) no operands had 0 or 5 as a unit digit (e.g., 40+65), (b) no digits were repeated within operands (e.g., 22+63), (c) the first operand was larger than the second operand in half the problems (e.g., 73+38) and vice versa in the other problems (e.g., 27+64), and (d) the sum of the unit digits was never equal to 10 (e.g., 26+64).

Two other sets of 16 problems each were tested under the one-strategy condition. These problems were the same as those tested under the two-strategy condition, but the order of operands was reversed (e.g., $31+58$ was in one of the first two sets of problems tested under the two-strategy condition and 58+31 was in one of the two sets of problems tested under the one-strategy condition). One of these two sets of 16 problems was solved with the MRD strategy and the other with the MRU strategy by half the participants, and the reverse for the other participants.

\subsection{Procedure}

Before encountering the experimental problems, children were told that they were going to do computational estimation. The computational estimation task was explained as giving an approximate answer to an arithmetic problem (e.g., 34+57) that is as close as possible to the correct answer without actually calculating the correct answer. They were told to use only two rounding strategies, the MRD or the MRU strategy. With the MRD strategy, participants had to round the first operand down and the second operand up to their closest decades (e.g., $30+60$ ). With the MRU strategy, participants had to round the first operand up and the second operand down to their closest decades (e.g., 40+50). A cue, which was presented above the problem, indicated which strategy participants had to use (i.e., OB for Down Up in Dutch served as a cue for the MRD strategy and BO for Up Down in Dutch served as a cue for the MRU strategy). Participants had to use each strategy on half of the problems, whereby for half of the problems each strategy was the "better" strategy (e.g., when participants had to use the MRD strategy on problems with the unit digit of the first operand smaller than 5 and that of the second operand larger than 5) and for the other half the "poorer" strategy (e.g., when participants had to use the MRD strategy on problems with the unit digit of the first operand larger than 5 and that of the second operand smaller than 5).

Before the experiment started in earnest, participants practiced on 12 problems to familiarize themselves with the two available strategies and with the procedure. Then, they solved two blocks of 16 problems each under the two-strategy condition in which the MRD or MRU strategy was randomly cued on each problem, with the constraint that participants had to switch strategy on two consecutive problems for half the items and to repeat the same strategy for the other items. Then, children solved two blocks of 16 problems each under the 
one-strategy condition where respectively the MRD and MRU strategy were applied on all 16 problems. The two-strategy condition was always presented first in order to avoid carry-over effects from repeatedly applying a particular strategy in the one-strategy condition on children's strategy execution in the two-strategy condition (Siegler \& Lemaire, 1997). The order in which the two strategies had to be applied in the one-strategy condition was counterbalanced across participants.

Each trial started with a 500-ms blank screen before a 400-ms fixation cross displayed at the center of the computer screen. Then, the problem was displayed and remained until participants' response. Children were asked to calculate out loud so as to be sure of which strategy they used. On each trial, the experimenter recorded children's response and strategy used. Following previous studies using this procedure (e.g., Lemaire \& Brun, 2014, 2016; Hinault, Lemaire, \& Phililips, 2016; Lemaire \& Hinault, 2014; Uittenhove \& Lemaire, 2012, 2013), timing of each response began when the problem appeared on the screen and ended when the experimenter pressed the left mouse button, the latter event occurring as soon as possible after the participant's responses.

Participants were individually tested in one session that lasted approximately 45 minutes. Between two blocks, participants were allowed a short break. The computational estimation task was run on a DELL Latitude laptop and was controlled by E-prime software (Schneider, Eschman, \& Zuccolotto, 2002), which recorded latencies to the millisecond.

\section{Results}

Mean correct solution latencies and percentages of errors (an error estimate was coded 1 if the estimated sum differed from the expected estimate given the cued strategy, as children always used the cued strategy) were analyzed with mixed-design ANOVAs, 2 (Group: fifth, seventh graders) x 2 (Condition: one-strategy, two-strategy) x 2 (Strategy: mixed rounding-down, mixed rounding-up), with repeated measures on the two last factors (see Table 1). In all results, unless otherwise noted, differences are significant to at least $p<.05$.

Seventh graders were faster than fifth graders (4935 ms vs. $\left.5756 \mathrm{~ms} ; F(1,70)=11.02, M S \mathrm{e}=4411337.0, \eta_{\mathrm{p}}^{2}=0.14\right)$. All children were faster in the one-strategy condition $(5094 \mathrm{~ms})$ than in the two-strategy condition $(5597 \mathrm{~ms}$; $\left.F(1,70)=59.02, M S \mathrm{e}=308942.0, \eta_{\mathrm{p}}^{2}=0.46\right)$ and executed the MRD-strategy $(5258 \mathrm{~ms})$ more quickly than the MRU-strategy $\left(5433 \mathrm{~ms} ; F(1,70)=11.51, M S \mathrm{e}=192379.8, \eta_{\mathrm{p}}^{2}=0.14\right)$. The Group $\mathrm{x}$ Condition interaction $\left(F(1,70)=5.49, M S e=308942.0, \eta_{\mathrm{p}}^{2}=0.07\right)$ revealed that the effect of condition was larger in fifth graders $(657 \mathrm{~ms})$ than in seventh graders $(350 \mathrm{~ms})$. The Condition $\mathrm{x}$ Strategy interaction was significant $(F(1,70)=28.10$, $\left.M S \mathrm{e}=181469.1, \eta_{\mathrm{p}}^{2}=0.29\right)$, and the Group $\mathrm{x}$ Condition $\mathrm{x}$ Strategy was marginally significant $(F(1,70)=3.34$, $\left.M S \mathrm{e}=181469.1, \eta_{\mathrm{p}}^{2}=0.05, p=.07\right)$.

Separate analyses in each age group revealed that the Condition $\mathrm{x}$ Strategy interaction was significant in fifth graders $\left(F(1,35)=18.65, M S e=247674.5, \eta_{\mathrm{p}}^{2}=0.35\right)$ and in seventh graders $(F(1,35)=9.50, M S \mathrm{e}=115263.6$, $\left.\eta_{\mathrm{p}}^{2}=0.21\right)$. Fifth graders were 531-ms $\left(F(1,35)=15.51, M S \mathrm{e}=327242.6, \eta_{\mathrm{p}}^{2}=0.31\right)$ faster with the MRD strategy than with the MRU strategy in the one-strategy condition and $185-\mathrm{ms}\left(F(1,35)=4.15, M S \mathrm{e}=147609.2, \eta_{\mathrm{p}}^{2}=0.11\right)$ faster with the MRU than with the MRD strategy in the two-strategy condition. Seventh graders were 352-ms $\left(F(1,35)=11.90, M S \mathrm{e}=187398.3, \eta_{\mathrm{p}}^{2}=0.25\right)$ faster with the MRD strategy than with the MRU strategy under the one-strategy condition but were equally fast with the MRD and MRU strategies in the two-strategy condition $(F<0)$.

Table 1. Mean solution latencies (in ms) and percentages of errors for each strategy in each group under two-strategy and one-strategy conditions

\begin{tabular}{|c|c|c|c|c|c|c|c|c|}
\hline \multirow{2}{*}{ Strategies } & \multicolumn{4}{|c|}{ Fifth Graders } & \multicolumn{4}{|c|}{ Seventh Graders } \\
\hline & One-Strategy & Two-Strategy & Means & Differences & One-Strategy & Two-Strategy & Means & Differences \\
\hline & \multicolumn{8}{|c|}{ Latencies (in ms) } \\
\hline MRD Strategy & 5163 & 6177 & 5670 & 1015 & 4584 & 5108 & 4846 & 524 \\
\hline MRU Strategy & 5694 & 5992 & 5843 & 299 & 4936 & 5111 & 5024 & 175 \\
\hline Means & 5428 & 6085 & 5756 & 657 & 4760 & 5110 & 4935 & 350 \\
\hline
\end{tabular}




\begin{tabular}{|c|c|c|c|c|c|c|c|c|}
\hline Differences & 531 & -185 & 173 & & 352 & 3 & 178 & \\
\hline & \multicolumn{8}{|c|}{ Percentages of Errors } \\
\hline MRD Strategy & 1.1 & 1.1 & 1.1 & 0.0 & 2.8 & 0.3 & 0.4 & -2.4 \\
\hline MRU Strategy & 0.5 & 2.4 & 2.6 & 1.9 & 0.0 & 0.0 & 0.0 & 0.0 \\
\hline Means & 0.8 & 1.8 & 1.3 & 1.0 & 0.3 & 0.2 & 0.2 & -0.1 \\
\hline Differences & -0.6 & 1.4 & 1.5 & & -2.8 & -0.3 & -0.4 & \\
\hline
\end{tabular}

Note. MRD: Mixed Rounding-Down strategy; MRU: Mixed Rounding-Up strategy.

As can be seen in Table 1, children were very accurate. They erred on average on less than $2 \%$ of the problems. Fifth graders made more errors than seventh graders $\left(1.8 \%\right.$ vs. $\left.0.2 \% ; F(1,70)=14.41, M S e=13.1, \eta^{2}=0.17\right)$, and all children tended to err more while executing the MRD strategy compared to the MRU strategy $(0.8 \%$ vs. $1.3 \%$, $\left.F(1,70)=3.17, M S \mathrm{e}=6.5, \eta_{\mathrm{p}}^{2}=0.04, p=.08\right)$. The Group x Strategy interaction $\left(F(1,70)=10.48, M S \mathrm{e}=6.5, \eta_{\mathrm{p}}^{2}=0.13\right)$ revealed that this strategy difference was larger in fifth graders $(1.5 \%)$ than in seventh graders $(-0.4 \%)$. No other effects came out significant $(F \mathbf{s}<1)$.

\section{General Discussion}

In this study, we investigated how strategy execution changes with children's age and the number of available strategies. Fifth and seventh graders accomplished computational estimation task in conditions where only one versus two strategies were available. To control for strategy selection, which strategy had to be executed was cued for each problem. Results showed that children were surprisingly accurate. Due to these very low error rates, the accuracy measure was most probably not sufficiently sensitive to detect reliable differences between the one- and the two-strategy condition. With respect to speed, we found that both groups of children were faster under the one-strategy condition than under the two-strategy condition, and that group-related differences were larger under the two-strategy condition. Also, differences in speed tended to vary as a function of the number of strategies, and this strategy difference was largest in younger children. These findings have implications to further our understanding of strategy execution in arithmetic and in other cognitive domains, as well as of age-related differences in children's performance during cognitive development.

Although in both two- and one-strategy conditions participants did not have to select which strategy to use on each problem, the two-strategy condition differed from the one-strategy condition in several respects. First, the two available strategies were maintained active in the two-strategy condition, as either of them could be unpredictably cued on each problem. Such active maintenance may have consumed resources unavailable for most efficient execution of the cued strategy. In contrast, all processing resources were available for strategy execution in the one-strategy condition. Also, when participants encoded the to-be-executed strategy and the problem, they had to (at least partially) inhibit the irrelevant strategy and activate procedures of the cued strategy before executing them. Such inhibitory processes are not involved in the one-strategy condition. Finally, switching strategies across successive problems may have used resources that could not be used for strategy execution. Note though that we found no strategy switch costs, as seen in comparable latencies when children used the same strategy versus different strategies on two successive problems (5637 ms vs. $5528 \mathrm{~ms}, F<1)$. This lack of strategy switch costs contrasts with previous findings (e.g., Lemaire \& Leacheur, 2010; Lemaire \& Brun, 2014). Although it is possible that switching between strategies incurs no switching costs, in contrast to what has been found previously, it is also possible that switching between strategies incurred costs in this study but durations of switching processes were absorbed by durations of other processes involved during strategy execution (note that average solution times were over $4900 \mathrm{~ms}$ ). Nevertheless, a number of differences between two- and one-strategy conditions may have contributed to strategies being executed more slowly under the two-strategy condition.

Interestingly, we found that relative strategy performance differed across two- and one-strategy conditions. Children were 441-ms faster with the MRD than with the MRU strategy under the one-strategy condition and equally fast with both strategies under the two-strategy condition. MRD is easier than MRU, possibly because once the first operand is rounded down, this rounded operand is stored in working memory before adding it to the second operand. Executing MRU involves first rounding the second operand up and storing this first rounded operand in working memory, then rounding the second operand down while maintaining the first operand in 
working memory, and finally adding both rounded operands. Executing MRD involves first rounding first operand down and storing this rounded operand in working memory, then rounding second operand up, and finally adding both rounded operands. In other words, adding one rounded operand to the other operand takes more time if the rounding operation is carried out after encoding the first operand and temporarily storing it in working memory than if the rounding operation is first carried out before adding the second operand to this rounded operand.

Differences in strategy performance under the one-strategy condition and the lack of such differences in the two-strategy condition suggest that one-strategy condition yields better assessment of strategy execution (i.e., uncontaminated by other factors). Indeed, in the two-strategy condition, although relative strategy performance is not contaminated by strategy distribution and strategy selection (as both strategies are used by all participants on an equal number of problems), differences in strategy performance may have been absorbed by extra-time required to manage two strategies as is suggested by longer latencies in the two-strategy condition than in the one-strategy condition. Thus, as already argued by Siegler and Lemaire (1997) and many others since, when we want to assess relative difficulty of strategy execution and compare strategy performance, it is best to test participants under a condition in which all participants have to execute the same strategy on all problems, and to do this for all available strategies.

The final set of findings of interest in this experiment concerns age-related differences in children's performance. It is possible that these differences could partially be explained by age-related differences in experience with and skills underlying these strategies. However, as these age-related differences were modulated by the type of strategies, other more domain-general factors, such as cognitive resources, might contribute to these differences as well. Age-related differences in performance were larger under the two-strategy condition than under the one-strategy condition, as increased latencies from the one- to the two-strategy condition were larger in younger children. Again managing two strategies incurred more cognitive resources and as young children have fewer resources available, they increased their latencies in the two-strategy condition relative to the one-strategy condition to a larger extent than older children.

Both fifth and seventh graders were faster with the MRD strategy than with the MRU strategy. However, this strategy difference was found only in the one-strategy condition where it was larger in third than in fifth graders. This reflects typical larger age differences on the more difficult strategy that has been found in a number of cognitive domains (see Siegler, 1996). As the harder strategy requires more processing resources to execute and younger children have fewer resources available, they need more time to execute the harder strategy.

Even though previous studies have already demonstrated that the number of strategies that individuals are using is related to their executive resources (Ardiale et al., 2012; Hodzik \& Lemaire, 2011), future studies should examine the degree to which the effects observed in the present study could be attributed to children's measures of executive functions, like inhibition and shifting capacities, as well as working-memory. This approach will provide a direct test of the extent to which the number of strategies in someone's repertoire consumes executive resources and working-memory capacity.

The present findings have some potential educational implications as it is shown that the number of available strategies negatively affects children's arithmetic performance and that this effect becomes smaller with age. Although accuracy is a more important performance measure in education contexts, and we observed this effect only on children's latencies and not on their error rates, it cannot be ruled out that similar effects would occur on children's accuracy as well. Indeed, as mentioned earlier, children were surprisingly very accurate. Including more difficult problems in future work might exclude such ceiling effects and reveal similar effects on accuracy. In that case, it might be recommendable to instruct and let practice young children one strategy at a time, as to spare their cognitive resources and working-memory capacity. At a later age, when children's executive functions and working-memory capacity have improved, one might consider teaching multiple strategies at the same time (see also Jitendra et al., 2007).

The present effects of the number of strategies have important theoretical implications to further our understanding of how children execute strategies and age-related differences therein. Formal models of strategies (e.g., Lovett \& Anderson, 1996, ACT-R model; Lovett \& Schunn, 1999, RCCL model; Payne et al., 1993, adaptive decision maker model; Rieskamp \& Otto, 2006, SSL model; or Siegler \& Arraya, 2005, SCADS* model) share core assumptions regarding how participants execute strategies on each problem. For example, all models proposed that strategy performance depends on the number and difficulty of the different processing steps within a strategy. These models also assume that how each procedure within a strategy is executed depends on problem, situation, 
and participants characteristics. Finally, these models assume that age-related changes in children's speed of strategy execution involves an increase in the speed of triggering and executing the different component processes within a strategy. In other words, strategy execution on a given problem and age-related changes in strategy execution are, according to these models, independent of the number of strategies that are used across all problems in a given task. The effects of the number of strategies found here suggest that strategy execution and age-related changes in how children execute strategies are also influenced by processes that enable managing several strategies. Such processes involve working-memory and executive control processes (e.g., inhibition, switching). Assumptions of current models of strategies could be augmented to include assumptions regarding the role of these processes during strategy execution. Moreover, additional assumptions in current models of strategy selection and execution could computationally specify how relative strategy performance becomes more and more independent of the number of strategies being available for solving the different problems in a given task and, more generally, how developmental mechanisms enable children to more and more efficiently manage several strategies.

\section{Acknowledgments}

This work was supported by a grant from La Fondation de France to PL, under Grant \# BLAN-1912-01 to PL and the grant C16/16/001 of the Research Fund KU Leuven to KL. We would like to thank Katrien Franssen and Helena Van Hoof for their help during data collection.

\section{References}

Ardiale, E., Hodzik, S., \& Lemaire, P. (2012). Aging and strategy switch costs: A study in arithmetic problem solving. L'Année Psychologique, 112, 345-360. https://doi.org/10.4074/S0003503312003028

Campbell, J. I. D. (2005). Handbook of mathematical cognition. New York: Psychology Press.

Cohen Kadosh, R., \& Dowker, A. (2015). The Oxford handbook of numerical cognition. New York: Oxford University Press.

Dowker, A. (1997). Young children's addition estimates. Mathematical Cognition, 3(2), 141-154. https://doi.org/10.1080/135467997387452

Dowker, A., Flood, A., Griffiths, H., Harris, L., \& Hook, L. (1996). Estimation strategies of four groups. Mathematical Cognition, 2(2), 113-135. https://doi.org/10.1080/135467996387499

Geary, D. C. (1994). Children's mathematical development. Washington, DC: American Psychological Association.

Hinault, T., Lemaire, P., \& Phillips, N. (2016). Aging and sequential modulations of poorer-strategy effects: An EEG study in arithmetic problem solving. Brain Research, 1630, 144-158. https://doi.org/10.1016/j.brainres.2015.10.057

Hodzik, S., \& Lemaire, P. (2011). Inhibition and shifting capacities mediate adults' age-related differences in strategy selection and repertoire. Acta Psychologica, 137, 335-344. https://doi.org/10.1016/j.actpsy.2011.04.002

Jitendra, A. K., Griffin, C. C., Haria, P., Leh, J., Adams, A., \& Kaduvettoor, A. (2007). A comparison of single and multiple strategy instruction on third-grade students' mathematical problem solving. Journal of Educational Psychology, 99(1), 115-127. https://doi.org/10.1037/0022-0663.99.1.115

LeFevre, J., Greenham, S. L., \& Waheed, N. (1993). The development of procedural and conceptual knowledge in computational estimation. Cognition and Instruction, 11(2), 95-132. https://doi.org/10.1207/s1532690xci1102_1

Lemaire, P., \& Brun, F. (2014). Effects of strategy sequences and response-stimulus intervals on children's strategy selection and strategy execution: A study in computational estimation. Psychological Research, 78(4), 506-519. https://doi.org/10.1007/s00426-013-0501-0

Lemaire, P., \& Brun, F. (2016). Age-related differences in children's strategy repetition: A study in arithmetic. Journal of Experimental Child Psychology, 150, 227-240. https://doi.org/10.1016/j.jecp.2016.05.014

Lemaire, P., \& Hinault, T. (2014). Age-related differences in sequential modulations of poorer-strategy effects: A study in arithmetic problem solving. Experimental Psychology, 61(4), 253-262. https://doi.org/10.1027/1618-3169/a000244 
Lemaire, P., \& Lecacheur, M. (2002). Children's strategies in computational estimation. Journal of Experimental Child Psychology, 82, 281-304. https://doi.org/10.1016/S0022-0965(02)00107-8

Lemaire, P., \& Lecacheur, M. (2010). Strategy switch costs in arithmetic problem solving. Memory \& Cognition, 38(3), 322-332. https://doi.org/10.3758/MC.38.3.322

Lemaire, P., \& Lecacheur, M. (2011). Age-related changes in children's executive functions and strategy selection: A study in computational estimation. Cognitive Development, 26(3), 282-294. https://doi.org/10.1016/j.cogdev.2011.01.002

Lemaire, P., \& Reder, L. (1999). What affects strategy selection in arithmetic? An example of parity and five effects on product verification. Memory \& Cognition, 22, 364-382. https://doi.org/10.3758/BF03211420

Lovett, M. C., \& Anderson, J. R. (1996). History of success and current context in problem solving: Combined influences on operator selection. Cognitive Psychology, 31(2), 168-217. https://doi.org/10.1006/cogp.1996.0016

Lovett, M. C., \& Schunn, C. D. (1999). Task representations, strategy variability, and base-rate neglect. Journal of Experimental Psychology: General, 128(2), 107-130. https://doi.org/10.1037/0096-3445.128.2.107

Marewski, J. N., \& Link, D. (2014). Strategy selection: An introduction to the modeling challenge. WIREs Cognitive Sciences, 5, 39-59. https://doi.org/10.1002/wcs.1265

Payne, J. W., Bettman, J. R., \& Johnson, E. J. (1993). The adaptive decision maker. New York: Cambridge University Press. https://doi.org/10.1017/CBO9781139173933

Reitan, R., \& Wolfson, D. (1992). Neuropsychological evaluation of older children. Tucson, AZ: Neuropsychology Press.

Reys, R., Bestgen, B., Rybolt, J., \& Wyatt, J. (1982). Processes used by good computational estimators. Journal for Research in Mathematics Education, 12(3), 183-201. https://doi.org/10.2307/748555

Rieskamp, J., \& Otto, P. E. (2006). SSL: A theory of how people learn to select strategies. Journal of Experimental Psychology: General, 135(2), 207-236. https://doi.org/10.1037/0096-3445.135.2.207

Schneider, W., Eschman, A., \& Zuccolotto, A. (2002). E-Prime User's Guide. Pittsburgh, PA: Psychology Software Tools Inc.

Siegler, R. S. (1996). Emerging minds. New York: Oxford University Press.

Siegler, R. S. (2007). Cognitive variability. Developmental Science, 10, 104-209. https://doi.org/10.1111/j.1467-7687.2007.00571.x

Siegler, R. S., \& Arraya, R. (2005). A computational model of conscious and unconscious strategy discovery. In R. V. Kail (Ed.), Advances in child development and behaviour (Vol. 33, pp. 1-42). Oxford, UK: Elsevier. https://doi.org/10.1016/s0065-2407(05)80003-5

Siegler, R. S., \& Lemaire, P. (1997). Older and younger adults' strategy choices in multiplication: Testing predictions of ASCM using the choice/no-choice method. Journal of Experimental Psychology: General, 126, 71-92. https://doi.org/10.1037/0096-3445.126.1.71

Sowder, J. T., \& Markovits, Z. (1990). Relative and absolute error in computational estimation. In G. Booker, P. Cobb, \& T. N. de Mendicuti (Eds.), Proceedings of the Fourteenth Psychology of Mathematics Education Conference (pp. 321-328). Mexico.

Uittenhove, K., \& Lemaire, P. (2012). Sequential difficulty effects during strategy execution: A study in arithmetic. Experimental Psychology, 59(5), 295-301. https://doi.org/10.1027/1618-3169/a000157

Uittenhove, K., \& Lemaire, P. (2013). Strategy sequential difficulty effects vary with working-memory and response-stimulus intervals: A study in arithmetic. Acta Psychologica, 143, 113-118. https://doi.org/10.1016/j.actpsy.2013.02.007

\section{Copyrights}

Copyright for this article is retained by the author(s), with first publication rights granted to the journal.

This is an open-access article distributed under the terms and conditions of the Creative Commons Attribution license (http://creativecommons.org/licenses/by/4.0/). 